\title{
Novel mutations of EVER1/TMC6 gene in a Japanese patient with epidermodysplasia verruciformis
}

Received: 25 November 2003 / Accepted: 31 January 2003/Published online: 23 March 2004

(C) The Japan Society of Human Genetics and Springer-Verlag 2004

\begin{abstract}
Germline mutations of the EVER1/TMC6 gene are associated with epidermodysplasia verruciformis (EV), which is characterized by an abnormal susceptibility to human papillomaviruses that were considered to be innocuous for the general population. In this study, we have employed polymerase chain reaction and DNA sequencing analysis to characterize the EVERI gene in a 65-year-old Japanese EV patient. Direct sequence analyses resulted in the identification of two novel mutations. One nonsense mutation consisting of a $(\mathrm{C}>\mathrm{A})$ transversion at nucleotide 744 in exon 8 in one EVER1 allele resulted in the introduction of a premature termination codon (Y248X). Another mutation was identified in the splice acceptor site of intron 8 (892-2, IVS8-2, A $>\mathrm{T}$ ) in another allele. This is the second report of EVER1/TMC6 mutations in EV.
\end{abstract}

Keywords Epidermodysplasia verruciformis .

EVER1/TMC6 - Mutation

\section{Introduction}

Epidermodysplasia verruciformis (EV) (OMIM\#226400) is a rare autosomal recessive disorder characterized by an abnormal susceptibility to infection by specific human papillomaviruses (HPVs) and an apparently increased risk of skin cancer. Carcinomas develop particularly in sun-exposed skin and are usually associated with HPV5 or HPV8. EV thus represents an unusual condition in which HPV infection, inherited predisposition, and exposure to the sun all play a part and a model system, which provides us an opportunity

G. Tate $(\bowtie) \cdot$ T. Suzuki $\cdot$ K. Kishimoto $\cdot$ T. Mitsuya

Department of Surgical Pathology,

Showa University Fujigaoka Hospital,

1-30 Fujigaoka, Aoba-ku, Yokohama 227-8501, Japan

E-mail: six10352@super.win.ne.jp

Tel.: + 81-45-9746383

Fax: $+81-45-9726242$ to analyze the genetic basis for the susceptibility to HPV infections (Lutzner and Blanchet-Bardon 1987). Ramoz et al. (2002) studied two Algerian and two Colombian consanguineous EV families and identified that EVERI/ $T M C 6$ and EVER2/TMC8 genes are responsible for the EV. EVER1 (19 exons) and EVER2 (15 exons) genes are in the opposite orientation from the first in frame initiation codon and are separated by $4,732 \mathrm{bp}$. Two kinds of EVER1 transcripts, $2.8 \mathrm{~Kb}$ in full length and $1.7 \mathrm{~Kb}$ that skipped from exon 11 to exon 18, are reported (Ramoz et al. 2002; Keresztes et al. 2003; Kurima et al. 2003). The gene product, EVER1, has features of integral membrane proteins and is localized in the endoplasmic reticulum (Ramoz et al. 2002). Here we report novel EVER1 mutations in an EV patient.

\section{Case report}

The patient was a 65 -year-old Japanese woman. She frequently suffered from flat warts beginning when she was a teenager. Excisions of Bowen disease in the hand were carried out three times when she was 51, 52, and 64, and a gastrectomy was performed because of gastric cancer when she was 53 . These surgeries were performed at another hospital. Diagnosis of EV was made when she was in her twenties. Surgery was carried out at this hospital because multiple skin cancers were found on her face. Histological diagnosis of these excised skin cancers showed squamous cell carcinoma and Bowen disease (data not shown). Her parents had died of heart disease and showed no sign of EV. They were not consanguineous. Three individuals are affected with EV among her five brothers and one sister. The family history was taken from the proband only, and the authors did not examined it. Informed consent for genetic analysis was obtained from the patient and normal individuals. However, genetic analyses of her brothers and her daughter were unavailable because they refused to provide the blood.

\section{Methods}

Genomic DNA was prepared from peripheral blood leukocytes by standard methods using phenol/chloroform extraction and ethanol precipitation. The exons and the intron/exon boundaries in the EVER1 gene were analyzed by polymerase chain reaction (PCR) 
and direct sequencing of the PCR products (Tate et al. 2003). The primers used for amplification of the genomic DNA were as follows: EVER1, ex8-1, 5'-TGG CGC TAC GCC CTG AAG CGC ATC-3', EVER1, ex8R, 5'-GTA CCA GGG TAG CAG AAG ACA GG-3', EVER1, ex9F, 5'-CGT GGG GAA AGT CCC TGA CTC GGC-3', EVER1, ex9R, 5'-GAG TGG AGC TCG GAG CCT GGG TC-3'. Primer sequences for other exons are available on request. Amplified DNA fragments were recovered from a lowmelting-temperature agarose gel and used for the sequencing analysis. We sequenced the PCR products directly by using a DNA sequencing system (model 377; Applied Biosystems). The direct sequencing was performed in both directions, and the mutation analysis was repeated independently. Nucleotide numbering is based on Genbank sequence AY099356 (EVER1 $2.8 \mathrm{~Kb}$ cDNA), AY099357 (EVER1 1.7 Kb cDNA) and NT010641 (EVER1 genomic DNA; gi: 29797789). PCR fragments amplified by a pair of primers, EVER1, ex8-1 and ex9R were cloned by pGEM-T easy vector systems according to the manufacturer's technical protocol (Promega, Madison, WI, USA) and sequenced by primer extension methods.

\section{Results}

As shown in Fig. 1, mutational analysis of the EVERI gene using primers EVER1, ex8-1, and ex9R revealed a heterozygous single nucleotide transversion from $\mathrm{C}$ to $\mathrm{A}$ at nucleotide 744 , which resulted in the introduction of a premature termination codon in exon 8 (Y248X). In addition, we found another heterozygous single nucleotide transversion in the splice acceptor site of intron 8 (892-2, IVS8-2, A > T) (Fig. 2). Taken together with the

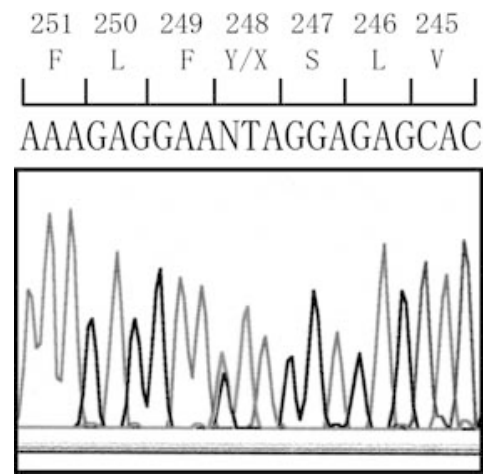

Fig. 1 A nonsense mutation in exon 8 of the EVERl gene in the epidermodysplasia verruciformis (EV) patient. Genomic DNA sequence analysis of exon 8 revealed a heterozygous transversion at nucleotide 744 in exon 8 , which is shown by $N$, resulting in a premature termination codon $(744 \mathrm{C}>\mathrm{A}) . \quad N$ indicates the overlapping waves $\mathrm{G}$ and $\mathrm{T}$. The reverse-complementary nucleotide sequence is shown first report that described that $E V E R I$ is the responsible gene of EV (Ramoz et al. 2002), sequencing data obtained from 50 normal individuals showed no nucleotide transversions in these nucleotides (data not shown), indicating that these two transversions are not polymorphisms but mutations.

To determine whether two mutations found in this study locate on the same allele or separately on the different alleles, PCR fragments amplified with a pair of primers EVER1, ex8-1 and EVER1, ex9R were cloned with pGEM-T easy vector and sequenced. The results of the sequence by primer extension indicated that two mutations located on the different alleles, consistent with the autosomal recessive trait (data not shown).

\section{Discussion}

Four kinds of mutations in the EVER1 and EVER2 genes have already been reported in EV patients of two Algerian and two Colombian consanguineous families (Ramoz et al. 2002). As shown in Table 1, three of four EVER1 mutations are nonsense mutations, and one is a splicing site mutation. Carcinomas develop particularly in sun-exposed skin and are usually associated with HPV 5 or HPV8 in EV. In this study, however, E6 genes of HPV5 and HPV8 were undetected in the skin cancer

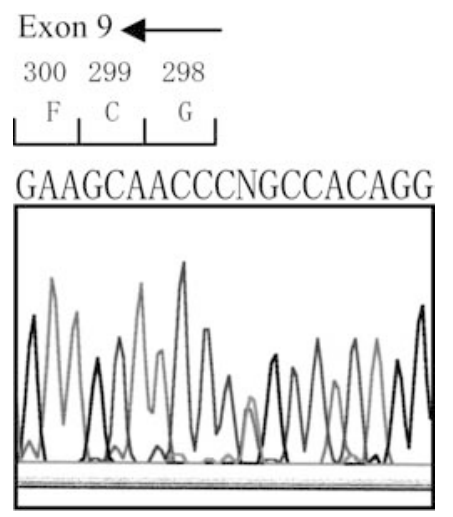

Fig. 2 A splicing mutation in intron 8 of the EVER1 gene in the epidermodysplasia verruciformis (EV) patient. Genomic DNA sequence analysis at the splicing acceptor site of intron 8 revealed a heterozygous $\mathrm{A}$ to $\mathrm{T}$ substitution $(892-2$, IVS8-2 $\mathrm{A}>\mathrm{T}) . N$ indicates the overlapping waves $\mathrm{A}$ and $\mathrm{T}$. The nucleotide $\mathrm{A}$ occurs in the wild-type sequence and the nucleotide $\mathrm{T}$ in the mutated sequence. The reverse-complementary nucleotide sequences and amino acid numbers are shown

Table 1 Summary of EVER1 mutations in EV

\begin{tabular}{|c|c|c|c|c|c|c|}
\hline Mutation & Splice mutation & Splice site & Exon & $\operatorname{IVS}^{\mathrm{a}}$ & Amino acid & Source \\
\hline $280(\mathrm{C}>\mathrm{T})^{\mathrm{b}}$ & No & \multirow{3}{*}{ Acceptor } & 5 & \multirow{3}{*}{8} & R94X & Ramoz et al. (2002) \\
\hline $744(\mathrm{C}>\mathrm{A})$ & No & & 8 & & Y248X & Present study \\
\hline $1726(\mathrm{G}>\mathrm{T})$ & No & & 14 & & E576X & Ramoz et al. (2002) \\
\hline
\end{tabular}

${ }^{\mathrm{a}}$ IVS, intervening sequence

${ }^{\mathrm{b}}$ Nucleotide position 1 is the A in the ATG translation initiation codon in the EVER1 cDNA sequence 
by PCR (data not shown). An EV patient in this case suffered from gastric cancer in addition to skin cancer. Her brother with EV also suffered from gastric cancer, and her sister with EV suffered from bilateral breast cancer, indicating that EVERI mutations may be involved in cancer predisposition not only in skin but also in other organs.

The function of the EVER1 molecule is unknown, although it is reported that EVER1 is localized in the endoplasmic reticulum (Ramoz et al. 2002). Therefore, it remains to be discovered why HPV infection caused skin cancer in the EV patient and which steps, such as antigen presentations against HPV, cytokine secretions, or cytotoxic T-lymphocyte inductions, are disturbed. In conclusion, we report here the first EVERI mutation in a Japanese patient with EV.

Acknowledgements We thank the patient for making this study possible. This work was supported in part by the High-Technology Center Project from the Ministry of Education, Science, Sports, Culture, and Technology of Japan (GT).

\section{References}

Keresztes G, Mutai H, Heller S (2003) TMC and EVER genes belong to a large novel family encoding transmembrane proteins. BMC Genomics 4:24

Kurima K, Yang Y, Sorber K, Griffith AJ (2003) Characterization of the transmembrane channel-like (TMC) gene family: functional clues from hearing loss and epidermodysplasia verruciformis small star, filled. Genomics 82:300-308

Lutzner MA, Blanchet-Bardon C (1987) Epidermodysplasia verruciformis (Lewandowsky-Lutz syndrome). In: Fitzpatrick TB, Eisen AZ, Wolff K, Freedberg IM, Austen KF (eds) Dermatology in general medicine. McGraw-Hill, Inc., New York, pp 2364-2372

Ramoz N, Rueda L-A, Bouadjar B, Montoya L-S, Orth G, Favre M (2002) Mutations in two adjacent novel genes are associated with epidermodysplasia verruciformis. Nat Genet 32:579-581

Tate G, Li M, Suzuki T, Mitsuya T (2003) A new germline mutation of the $P T C H$ gene in a Japanese patient with nevoid basal cell carcinoma syndrome associated with meningioma. Jpn J Clin Oncol 33:47-50 